\title{
Covid-19 Delirium in older inpatients with COVID-19: impact on service provision
}

\author{
Authors: Benjamin Low, ${ }^{A}$ Nicole QQ Xian, ${ }^{B}$ Isabelle Brooks, ${ }^{B}$ Abigail Heller, ${ }^{A}$ Batol Daher ${ }^{A}$ and Melanie Dani ${ }^{\mathrm{C}}$
}

Delirium is a common clinical manifestation of SARS-CoV-2 (COVID-19) in older inpatients. We assessed the prevalence of delirium in inpatients aged over 65 years with confirmed COVID-19 infection to identify its clinical correlations and association with in-hospital mortality and admission duration. Data were extracted retrospectively from electronic health records. The prevalence of delirium was found to be $23.9 \%$ (158 out of 662 patients). Factors associated with delirium included older age, dementia (including cases of suspected dementia), frailty and concurrent infection. Delirium was not associated with higher mortality. Admission duration was approximately 1.5 times longer in patients who experienced delirium (median 14 days; interquartile range (IQR) 8-30) compared with those who did not (median 9 days; IQR 5-17; $\mathbf{p}<0.001$ ). We confirmed that delirium is common in older inpatients with COVID-19 and has significant implications for patient care and planning services and rehabilitation.

KEYWORDS: delirium, COVID-19, older people

DOI: $10.7861 /$ fhj.2021-0017

\section{Introduction}

Delirium, defined as an acute disorder of attention and cognition, is increasingly recognised as a significant neuropsychiatric component of SARS-CoV-2 infection, either as a presenting symptom or a complication experienced during admission. ${ }^{1-3}$ At present, there is a lack of systematic assessment for delirium in COVID-19 management guidelines, lack of awareness among healthcare professionals that delirium can be an atypical presenting feature and an underappreciation of the poor prognosis that delirium confers in older patients. ${ }^{4,5}$

Recent studies have improved our understanding of the prevalence, incidence and clinical consequences of delirium in patients with SARS-CoV-2 infection. ${ }^{6}$ The prevalence in hospitalised patients has been described as around $20 \%-30 \%$,

Authors: ${ }^{\text {A }}$ senior house officer, Imperial College Healthcare NHS Trust, London, UK; ${ }^{B}$ foundation year-1 doctor, Imperial College Healthcare NHS Trust, London, UK; ${ }^{C}$ consultant geriatrician, Imperial College Healthcare NHS Trust, London, UK and Imperial College London, London, UK with rates reaching $60 \%-70 \%$ in severe illness in all ages. ${ }^{1,2}$ It is not an incidental finding; medium-term follow-up studies have shown poorer physical function at 4 weeks post-diagnosis of delirium in inpatients with SARS-CoV-2 infection. ${ }^{\text {? }}$

The aim of this retrospective case notes review was to assess delirium prevalence in a large and diverse population of older patients hospitalised with COVID-19 in north-west London to identify factors associated with its presence and to determine the implications on clinical outcome including in-hospital mortality and admission duration. This was part of a wider service evaluation of delirium assessment in an acute medical setting.

\section{Methods}

We performed a retrospective analysis of case records for 662 patients with a diagnosis of SARS-CoV-2 infection confirmed by reverse transcriptase-polymerase chain reaction assay for SARS-CoV-2 RNA from combined oropharyngeal/nasopharyngeal swab samples. All patients aged 65 years and older who attended Imperial College Healthcare NHS Trust consecutively for nonelective admission between 01 March 2020 and 09 May 2020 were included. There were no exclusion criteria. The majority of patients were admitted to general level 1 COVID-19 surge wards staffed by healthcare professionals redeployed from various specialties. None of the patients in this cohort were admitted to intensive care or high-dependency areas.

From each clinical record, we retrieved data on demographics, past medical history, concurrent infection or delirium, Rockwood clinical frailty score (CFS), social circumstances, admission duration and inpatient mortality. ${ }^{8}$ Delirium was confirmed following a detailed review of all medical, nursing and therapist notes for validated assessments (including 4AT and Confusion Assessment Method (CAM)) or presence of keywords related to delirium (such as confusion, disorientation, agitation and drowsiness) and with evidence of acute change from baseline attention and/or cognition. ${ }^{9,10}$ Pre-disease CFS was extracted directly from patient notes or retrospectively calculated based on the documented social and functional history. Suspected cases of dementia were determined by clinical suspicion from the medical team with therapist support and collateral history where obtainable. Concurrent infection was defined by documentation of clinical diagnosis of concurrent or alternative infection at any point from admission to discharge. Mortality during admission with positive SARS-CoV-2 result was confirmed by both the doctor's documentation of death verification and uploaded medical certificate of cause of death (MCCD). Baseline characteristics were 


\begin{tabular}{|c|c|c|c|c|}
\hline & $\begin{array}{l}\text { Patients without } \\
\text { delirium, } n=504\end{array}$ & $\begin{array}{l}\text { Patients with } \\
\text { delirium, } n=158\end{array}$ & p-value & $\begin{array}{l}\text { Adjusted OR } \\
(95 \% \mathrm{CI})\end{array}$ \\
\hline Age, median years (IQR) & $77(71-84)$ & $82(76-88)$ & $p<0.001$ & $1.05(1.02-1.07)$ \\
\hline Male, n (\%) & $286(56.7)$ & $94(59.5)$ & & \\
\hline \multicolumn{5}{|l|}{ Ethnicity, n (\%) } \\
\hline White & $185(36.7)$ & 75 (47.5) & & \\
\hline Asian & $51(10.1)$ & $12(7.6)$ & & \\
\hline Black & $72(14.3)$ & $21(13.3)$ & & \\
\hline Other & $179(35.5)$ & $47(29.7)$ & & \\
\hline Not stated & $17(3.4)$ & $3(1.9)$ & & \\
\hline \multicolumn{5}{|l|}{ Medical history } \\
\hline Dementia (including suspected), n ( \% ) & $95(18.8)$ & $64(40.5)$ & $p<0.001$ & $1.92(1.24-2.99)$ \\
\hline Clinical frailty score $\geq 5, \mathrm{n}(\%)$ & $238(47.2)$ & $107(67.7)$ & $p<0.001$ & $1.55(1.01-2.38)$ \\
\hline Hypertension, n (\%) & $292(57.9)$ & $106(67.1)$ & $p<0.05$ & \\
\hline Diabetes mellitus, n (\%) & $194(38.5)$ & $66(41.8)$ & & \\
\hline Ischaemic heart disease, n (\%) & $113(22.4)$ & $37(23.4)$ & & \\
\hline Chronic kidney disease, n (\%) & $88(17.5)$ & $33(20.9)$ & & \\
\hline Stroke/TIA, n ( \% ) & $71(14.1)$ & $30(19.0)$ & & \\
\hline Chronic respiratory disease, $\mathrm{n}(\%)$ & $124(24.6)$ & $33(20.9)$ & & \\
\hline Cancer, n (\%) & $114(22.6)$ & $31(19.6)$ & & \\
\hline Polypharmacy, n (\%) & $327(64.9)$ & $109(69)$ & & \\
\hline \multicolumn{5}{|l|}{ Factors related to admission } \\
\hline Concurrent infection, n (\%) & $223(44.2)$ & $96(60.8)$ & $\mathrm{p}<0.001$ & $2.02(1.38-2.96)$ \\
\hline \multicolumn{5}{|l|}{ Clinical outcomes } \\
\hline Mortality, n (\%) & $213(42.3)$ & $57(36.1)$ & & \\
\hline Length of admission, median days (IQR) & $9(5-17)$ & $14(8-30)$ & $p<0.001$ & \\
\hline
\end{tabular}

$\mathrm{CI}=$ confidence interval; $\mathrm{IQR}=$ interquartile range; $\mathrm{OR}=$ odds ratio; $\mathrm{TIA}=$ transient ischaemic attack.

compared between individuals who developed delirium and those who did not using chi-squared and Mann-Whitney $U$ tests as appropriate followed by multivariable regression analysis.

\section{Results}

The study population included 662 patients (mean age $78.7 \pm 8.1$ years; 380 men) admitted with confirmed COVID-19 between 01 March 2020 and 09 May 2020. One-hundred and fiftyeight (23.9\%) patients were documented as having delirium on at least one occasion during admission. Table 1 compares demographics, past medical history and clinical outcomes between patients who developed delirium and those who did not. One-hundred and seven (16.1\%) patients underwent formal cognitive assessment; 55 (8.3\%) of these were 4 AT or CAM.

Factors associated with delirium included older age, dementia (including suspected cases), frailty and concurrent infection $(p<0.001$ for all). These factors remained significant after multivariable logistic regression analysis. Delirium was not associated with higher mortality in univariable analysis. Admission length was longer in patients who experienced delirium (median 14 days; interquartile range (IQR) 8-30) compared with those who did not (median 9 days; IQR 5-17; $p<0.001$ ).

\section{Discussion}

Delirium is an acute disorder of attention and cognition. It remains a common, serious, under-recognised and, often, fatal condition for older patients. ${ }^{5}$ Diagnosis requires a formal cognitive assessment and history of acute onset. The prevalence of delirium in our population was high $(23.9 \%)$ and reflected the numbers reported globally during the first wave of the pandemic., ${ }^{1,2}$ As may be expected, factors associated with delirium included older age, dementia, frailty and concurrent infection. In our cohort, delirium was not associated with higher mortality. This is surprising given the association with mortality demonstrated in early studies and with studies examining older people in non-COVID-19 settings. ${ }^{11-13}$ However, the link is not clear-cut and recent studies (including data published by the CovidCollab) suggest other confounding factors (such as older age and higher frailty scores) as better predictors. ${ }^{6,14}$ 
Our data show that delirium is associated with a significantly longer admission duration. There are few studies that focus on this, which is surprising given the concern about inpatient bed capacity during the first wave of the pandemic and the current pressures experienced across the country. Median length of hospital stay was approximately 1.5 times greater in patients who experienced delirium. The implications of this are significant and should be borne in mind when planning how to respond to service pressures from potential further waves of COVID-19 infection. In light of this and other recent studies demonstrating the negative outcomes from delirium, it is important that COVID-19 guidelines emphasise identification of at-risk patients and early detection. Recognising and addressing delirium early can significantly improve patient care, allowing the clinician to promptly introduce simple interventions to reduce the severity and the length of delirium, and alleviate patient distress.

\section{Limitations}

There are several factors which may have affected the accuracy of our data and should prompt caution in interpreting our results, largely as a consequence of the retrospective nature. Firstly, it is difficult to accurately determine the prevalence of delirium. Documentation and formal assessment were inconsistent and it is likely that there was a significant degree of underreporting in the context of the initial surge of cases when large patient numbers were admitted over a short time. Furthermore, those who presented in a critically unwell state and died shortly after admission may not have undergone any delirium assessment at all. Secondly, we acknowledge that retrospective calculation of the CFS is not validated. Thirdly, caution should be exercised in interpretation of 'suspected dementia'. While cases of suspected dementia were largely supported by collateral history, we acknowledge the inherent clinical error in making this assumption and recognise that a validated tool such as the IQCODE (Informant Questionnaire on Cognitive Decline in the Elderly) would have been a more reliable method to support diagnosis. Finally, when assessing for the incidence of delirium, we did not account for multiple episodes of delirium, rather, we only included the documented presence of delirium on at least one occasion. Measuring the effects of multiple episodes of delirium is an important future research question.

\section{Conclusion and future recommendations}

We found that delirium was highly prevalent in older inpatients with COVID-19 infection and was associated with significantly longer admission. This is critically important as we continue to manage service capacity and patient turnover in subsequent waves of the pandemic. We postulate that NHS services, which recognise delirium as a significant rate-limiting factor in patient turnover, would be better equipped to manage current pressures. Patients with delirium would benefit from alternative care pathways; for example, direct transfer from the emergency department to delirium-friendly wards with controlled sensory input and specially trained staff.

\section{References}

1 Mao L, Jin H, Wang M et al. Neurologic manifestations of hospitalized patients with coronavirus disease 2019 in Wuhan, China. JAMA Neurol 2020;77:683-90.

2 Helms J, Kremer S, Merdji $\mathrm{H}$ et al. Neurologic features in severe SARS-CoV-2 infection. N Engl J Med 2020;382:2268-70.

3 Zazzara MB, Penfold RS, Roberts AL et al. Probable delirium is a presenting symptom of COVID-19 in frail, older adults: a cohort study of 322 hospitalised and 535 community-based older adults. Age Ageing 2021;50:40-8.

4 O'Hanlon S, Inouye SK. Delirium: a missing piece in the COVID-19 pandemic puzzle. Age Ageing 2020;49:497-8.

5 Inouye SK, Westendorp RGJ, Saczynski JS. Delirium in elderly people. Lancet 2014;383:911-22.

6 Ticinesi A, Cerundolo N, Parise A et al. Delirium in COVID-19: epidemiology and clinical correlations in a large group of patients admitted to an academic hospital. Aging Clin Exp Res 2020;32: 2159-66.

7 Mcloughlin BC, Miles A, Webb TE et al. Functional and cognitive outcomes after COVID-19 delirium. Eur Geriatr Med 2020;11: 857-62.

8 Rockwood K, Song X, MacKnight C et al. A global clinical measure of fitness and frailty in elderly people. Can Med Assoc J 2005;173:489-95.

9 4AT: rapid clinical test for delirium. www.the4AT.com [Accessed 26 January 2021]

10 Inouye SK, van Dyck CH, Alessi CA et al. Clarifying confusion: the confusion assessment method. A new method for detection of delirium. Ann Intern Med 1990;113:941-8.

11 Garcez FB, Aliberti MJR, Poco PCE et al. Delirium and adverse outcomes in hospitalized patients with COVID-19. J Am Geriatr Soc 2020;68:2440-6.

12 Marengoni A, Zucchelli A, Grande G, Fratiglioni L, Rizzuto D. The impact of delirium on outcomes for older adults hospitalised with COVID-19. Age Ageing 2020;49:923-6.

13 Witlox ], Eurelings LSM, de Jonghe JFM et al. Delirium in elderly patients and the risk of postdischarge mortality, institutionalization, and dementia: a meta-analysis. JAMA 2010;304:443-51.

14 Geriatric Medicine Research Collaborative, Covid Collaborative, Welch C. Age and frailty are independently associated with increased COVID-19 mortality and increased care needs in survivors: results of an international multi-centre study. Age Ageing 2021;50:617-30.

15 Jorm AF. A short form of the Informant Questionnaire on Cognitive Decline in the Elderly (IQCODE): development and cross-validation. Psychol Med 1994;24:145-53.

Address for correspondence: Dr Melanie Dani, Imperial College Healthcare NHS Trust, Hammersmith Hospital, Du Cane Road, London W12 0HS, UK.

Email: melanie.dani@nhs.net 